\title{
ELECCIÓN DE CARRERA Y GÉNERO
}

\author{
Jairo M. Ruiz-Gutiérrez \\ Universidad Alfonso López Pumarejo (Colombia) \\ Lidia E. Santana-Vega \\ Universidad de La Laguna (España)
}

\begin{abstract}
"Hablar de género es reconocer que las relaciones entre las personas están mediadas por las construcciones sociales y culturales que se hacen en base al sexo biológico"
\end{abstract}

(Ministerio de Educación de Chile, 2015: 9)

\begin{abstract}
Resumen. En la actualidad las mujeres han venido posicionándose social y profesionalmente. Uno de los objetivos dentro del contexto escolar es conocer si el gnénero o los estereotipos de género influyen en la elección de carrera de los chicos y las chicas al finalizar el bachillerato. En nuestra investigación se plantearon los siguientes interrogantes: 1) ¿Qué piensan hacer los estudiantes cuando terminen su bachillerato? 2) ¿Cuáles son los motivos o intereses que los lleva a tomar su decisión o elección? 3) ¿Como incide el género en la toma de decisiones al final del bachillerato? En la investigación se llevó a cabo un estudio de carácter descriptivo en el que participaron 155 estudiantes, 87 hombres y 68 mujeres; se adaptó y aplicó el Cuestionario de Orientación Académico y Laboral II (COAL II), diseñado por el Grupo de Investigación en Orientación Educativa y Sociolaboral (GIOES) de la Universidad de La Laguna. Los análisis estadísticos muestran diferencias en la elección de carrera por razón de género, esto es, los chicos y las chicas se inclinan por estudios u ocupaciones que tradicionalmente han sido consideradas propias de cada sexo. Las chicas prefieren carreras donde predominan los valores de sensibilidad y altruismo, mientras los chicos se decantan por el poder y el prestigio como factores determinantes en la elección de carrera.
\end{abstract}

Palabras Clave: contexto escolar, elección de carrera, motivos personales, estereotipos de género.

\section{CAREER CHOICE AND GENDER}

\begin{abstract}
At present women have been positioning themselves socially and professionally. One of the objectives within the school context is to know if gender or gender stereotypes influence the choice of career that boys and girls do at the end of high school. In the research the following questions were raised: 1) what do students plan to do when they finish high school? 2) What are the motives or interests that lead them to make their decision or choice? 3) How does gender affect decision making at the end of high school? The research is part of a descriptive study, in which 155 students participated, 87 boys and 68 girls. For this research, the Questionnaire of Academic and Professional Guidance II (COAL II) designed by the Research Group on Educational Orientation and Sociolaboral (GIOES) of the University of La Laguna, was adapted and applied. Statistical analyzes show differences in career choice by gender. That is, boys and girls are
\end{abstract}


inclined toward studies or occupations that have traditionally been considered sex-specific. The girls prefer careers where the values of sensitivity and altruism predominate, while the boys opt for power and prestige as determining factors in the choice of career.

Key words: school context, career choice, personal motives, gender stereotypes.

\title{
ESCOLHA DE CARREIRA E GÊNERO
}

\begin{abstract}
Resumo. $\mathrm{Na}$ atualidade as mulheres têm vindo a posicionar-se socialmente e profissionalmente. Um dos objetivos dentro do contexto escolar é a de saber se o gênero ou os estereótipos de gênero influenciam as escolhas de carreira que fazem meninos e meninas a terminar o ensino médio. Para esta investigação as seguintes questões foram levantadas: 1) O que os alunos pensam quando terminar a sua escola? 2) Quais são os motivos ou interesses que os leva a fazer a sua decisão ou escolha? 3) Como o género no final do ensino médio de tomada de decisão? A pesquisa envolveu 155 estudantes, 87 homens e 68 mulheres. Para esta investigação foi adaptado e aplicado o Questionário de Orientação Acadêmico e de Trabalho II (COAL II), concebido pelo Grupo de Pesquisa em Educação e Sócio (GIOES) da Universidade de La Laguna. Análises estatísticas mostram diferenças na escolhas de carreira por gênero, ou seja, meninos e meninas estão inclinados a estudos ou ocupações que têm sido tradicionalmente considerados próprio de cada gênero. As meninas preferem carreiras onde os valores de sensibilidade e altruísmo predominam, enquanto os meninos optam pelo poder e pelo prestígio como fatores determinantes na escolha da carreira.
\end{abstract}

Palavras-chave: contexto escolar, escolha profissional, razões pessoais, estereótipos de gênero.

\section{Introducción}

La mujer tiende a reproducir los papeles tradicionales que ha jugado en la vida doméstica cuando se incorpora a la esfera pública y al mundo del trabajo. Por lo general las profesiones relacionadas con la sanidad, la educación y las tareas sociales tienen "nombre de mujer, y las profesiones vinculadas con la economía, la industria, la alta política y las relaciones exteriores son elegidas por los hombres. Rivière (2000) sostiene, con gran ironía por cierto, que tal vez cuando las mujeres demuestren que las cuentas de la cocina sirven para gestionar la globalización, quizá entonces tengan una mayor presencia en los asuntos económicos. La construcción de la igualdad habrá de estar regida por la lógica del mestizaje y no de la dominación. La lógica de la dominación ahoga el punto de vista de las mujeres y les atribuye un papel de subordinación en la esfera social y empobrece por igual a las dos mitades de la humanidad (Santana Vega, 2002). En la carrera de fondo por alcanzar la igualdad de oportunidades el papel del sistema educativo es esencial ya que puede contribuir a situar a la mujer en el mundo redescubriendo su historia y recuperando su voz perdida. Como sostiene Torres Santomé (1994), realmente desconocemos la historia de la mujer y por qué se la ha silenciado. Según este autor la preocupación por el mundo femenino debe seguir existiendo ya que en la sociedad actual se está produciendo una fuerte remasculinización y constantes ataques a la filosofía de la igualdad.

El fenómeno de la escolarización universal igualadora, y la idea que históricamente le acompaña de la cultura como algo valioso y digno de ser poseído por todos, ha favorecido la presencia de las mujeres en la vida cultural. Como sostiene Gimeno Sacristán (1998:13) los dos retos fundamentales de la modernidad en educación han sido "la aspiración al logro de la igualdad, por un lado, y el equilibrio difícil entre atender a la vez a sujetos contextualizados y a la cultura objetivada". El respeto a la diversidad avanza con 
paso firme en el inicio del siglo XXI, ya que integrar la diversidad permite, como señala Imbernón (1999), favorecer la convivencia de realidades plurales, de necesidades diferentes que van a enriquecer el espacio social del aula, del centro, pero también de la comunidad y de la sociedad en general. Un logro de capital importancia, y que ha relanzado el papel de la mujer en el mundo actual, es la consideración social positiva de que es objeto el rendimiento escolar de las alumnas. No siempre se ha mirado con buenos ojos el hecho de que las chicas se destaquen en las materias escolares y menos si donde sobresalen es en las disciplinas de ciencias. Las estadísticas se empeñan en mostrarnos que las cosas están cambiando en lo referente a los resultados académicos según el sexo.

El mejor "comportamiento" de las chicas ante los requerimientos de las tareas escolares es una tendencia cada vez más general. Pero este hecho, que viene a corregir una "expectativa perversa" según la cual las niñas tenían casi que excusarse por sus buenos rendimientos en las áreas de ciencias, no deja de ser preocupante para el normal desarrollo de los niños. Me explico, la presión social ejercida sobre los varones de que ellos tienen que labrarse un porvenir brillante porque serán los que forjen el futuro de la sociedad y serán el sustento de su familia, no ha desaparecido del todo. En una investigación realizada por López Sáez (1995) se constata que los chicos siguen poniendo como un motivo importante para elegir determinados estudios la creencia de que tal carrera le llevará a ganar más dinero. Aún hoy la elección de profesión sigue estando determinada por el sexo lo que, a su vez, condiciona la elección de estudios. Esto es fácilmente apreciable en la elección de los Ciclos Formativos. Las chicas muestran sobre todo preferencia por las familias profesionales de administración (auxiliar administrativo, ayudante de oficina, gestor de cobros y pagos...), sanitaria (auxiliar de enfermería, auxiliar bucodental, auxiliar de salud mental,..), imagen personal (esteticista, manicura...), peluquería, etc. Los chicos tienen especial predilección por las familias profesionales de naturaleza técnica: informática (analista-programador, gestor de proyectos...), fabricación mecánica (soldador, montador de productos metálicos estructurales...), electricidad-electrónica (instalador de líneas eléctricas, electricista industrial...). La incidencia de la variable sexo sobre la elección de estudios del alumnado de último curso de secundaria queda patente en una investigación realizado por Gaviria (1994) sobre una amplia muestra de chicos (3.519) y chicas (3.746). De los resultados derivados del estudio se llegó a la conclusión de que el sexo era más determinante que la clase social a la hora de condicionar los intereses profesionales. Mientras las chicas se decantaban por los estudios y profesiones relacionadas con las ciencias jurídicas, las ciencias humanas, de la salud, literarias, los chicos, por el contrario, lo hacían por las ramas de física y química.

Los mensajes trasmitidos a través de los medios de comunicación de masas, sobre todo los audiovisuales, ejercen una fuerte impronta sobre la elección de estudios y profesiones. Los anuncios publicitarios suelen estar cargados de los esquemas y estereotipos sociales tradicionales. Las series de TV dan visiones engañosas de los perfiles profesionales de ciertas profesiones, como es el caso de las abundantes series españolas y extrajeras, emitidas por diferentes canales, que giran en torno a la profesión médica. En una entrevista realizada a diferentes profesionales de la medicina, relataban la escasa adecuación de estos programas a lo que ellos viven en el día a día en su profesión. Los libros de texto tampoco están libres de estereotipos sexistas como apunta la investigación realizada por Blanco García (1999). En ella se constata que frente a 334 profesiones representadas por varones se ofrecen sólo 94 representadas por mujeres. Mientras los varones desempeñan ocupaciones de prestigio social, que suponen ejercicio del poder o contribución expresa al progreso de la sociedad, las mujeres tienen como marco prioritario de sus acciones las relaciones interpersonales. Según Castells (1998), la habilidad de las 
mujeres para relacionarse es una de sus armas más poderosas en su incorporación al mercado laboral. El hecho más que constatado de que el sexo sigue condicionando las elecciones vitales, motivó que la Confederación de Organizaciones Feministas a las elecciones al Parlamento Europeo el 13 de junio de 1999, plantease como objetivos prioritarios: la eliminación del material didáctico que reprodujera de forma explícita o implícita los estereotipos sexistas; la realización de labores de orientación vocacional y profesional no sesgada por razón de sexo y la adopción de las medidas oportunas para favorecer la igualdad de acceso de las chicas a los programas de garantía social. (Santana Vega, 2002)

Los esquemas de la igualdad están siendo introducidos con fórceps ya que tienen que abrirse paso entre la maraña de estereotipos sociales; y aún estamos lejos de que el principio de la igualdad entre los sexos traspase el tejido social en su conjunto. Los estereotipos de género están cambiando, las consignas tradicionales están cayendo en desuso pero las nuevas no terminan de implantarse, así que el desconcierto es la tónica común. En definitiva, todavía queda un trecho para alcanzar el viejo desideratum de la igualdad de oportunidades, pero ello no es óbice para que asumamos el compromiso de defender en todos los foros que sea posible la idea de que la cultura, la política, las profesiones no tienen sexo; lo que sí puede que tengan sexo son los prejuicios, los intereses o los convencionalismos sociales.

En los últimos años el gobierno colombiano ha hecho un gran esfuerzo por implementar políticas que dignifiquen el papel de la mujer en los distintos campos de la sociedad. No obstante, este problema social no puede reducirse para ser observada sólo desde el campo laboral. Es indudable que estas transformaciones tienen sus orígenes en la igualdad de oportunidades; hombres y mujeres han de tener las mismas oportunidades en el acceso a la cultura, a la formación, a la política... Actualmente Colombia es reconocida dentro de los países latinoamericanos por aumentar la participación de la mujer en los ciclos post-secundarios, como son los estudios técnicos y universitarios (Ministerio de Educación Nacional [MEN], 2013; Rico y Trucco, 2014). Sin embargo, dentro de esta paridad creciente existen estereotipos de género (Santana Vega, 2015). Los estereotipos "son representaciones simplificadas, esquematizadas, deformadas y socialmente compartidas sobre un grupo de personas, que conforman opiniones sobre rasgos atribuidos a dicho grupo, en ausencia de experiencias directas con tales individuos." (Sánchez García et al., 2013: 51). Aún hoy se ve a la mujer como un ser dependiente, emotivo, sensible, realizando labores del hogar, y proveedora de cuidados; mientras que el hombre se le percibe con otras cualidades bien distintas tales como independencia, fuerza, autonomía, dominio y competitividad.

El estereotipo escolar etiqueta a los chicos como mejores con los números y las actividades que demanden destrezas físicas como los deportes, mientras que las chicas son mejores en exteriorizar sus sentimientos ya sea en forma oral y/o escrita, por lo tanto, les va mejor en el aprendizaje de los idiomas. Estos rasgos sociales han hecho que los y las estudiantes al finalizar su ciclo de formación escolar opten por mantener este sesgo; es decir, los hombres elijan carreras que guardan una estrecha relación con las matemáticas como son las ingenierías y la arquitectura mientras las mujeres optan por estudios concernientes al área de la salud y a las humanidades (Informe del Programa Internacional para la Evaluación de Estudiantes [PISA], 2012; Organización de las Naciones Unidas para la Educación, la Ciencia y la Cultura [UNESCO], 2012). La elección de carrera está sujeta en gran parte a motivos e intereses de género, como son en las niñas el espíritu de servicio, el brindar protección y la compasión; en los niños el alto valor por lo material, como el dinero, el poder, y el prestigio. 
En los siguientes apartados señalaremos algunas dimensiones relevantes para el trabajo:

A. El interés o motivo personal se puede considerar como el motor interno que impulsa a cada ser humano para que luche con vehemencia por concretar sueños, alcanzar metas y vencer obstáculos. De acuerdo con Rodríguez Moreno (2014) dentro de los estudios psicológicos, a los adolescentes entre 15 y 20 años la planificación y la preparación para una profesión son dos de los intereses profesionales que más les preocupa. Así mismo, el prestigio, el dinero, la fama, el altruismo, entre otros, han sido objetos de numerosas investigaciones dentro de los procesos de orientación escolar (Santana Vega, Feliciano García y Jiménez Llanos, 2009, 2012, 2016; Alonso, 2010; Rodríguez Moreno, 2014), como factores determinantes para lo toma de decisiones por género. Con relación a los intereses personales, Rodríguez Moreno (2014) expresa que junto con los valores, las aptitudes, los logros y las experiencias influyen notablemente en las decisiones que se tomen durante toda la vida. El ser humano está llamado a reconocer en sí mismo sus intereses y motivaciones personales, la ambigüedad o limitación de estos puede provocar sentimientos de angustia, aislamiento o incomprensión (Santana Vega, Feliciano García y Cruz González, 2010; Mérida Serrano, Ramírez García, Corpas Reina, y González Alfaya, 2012). De igual forma, se debe considerar que a medida que el ser humano avanza en su proceso de crecimiento y evolución, algunas veces, va transformando sus intereses (Arancibia, Herrera, y Strasser, 2008; Sánchez y Álvarez, 2012; Rodríguez Moreno, 2014).

B. La elección de carrera afecta tanto la vida académica como la vida laboral del ser humano (Bisquerra Alzina, 1992; Barrero González, 2006). Si bien en la actualidad hay paridad de acceso para hombres y mujeres a las carreras profesionales, aún existen sesgos sexistas a la hora de la elección. "En la educación vocacional y en los programas de exploración de carreras, las mujeres se agrupan en las ocupaciones tradicionales femeninas" (Alonso, 2010: 795). Entre estas se encuentran catalogadas la docencia, y las ocupaciones de cuidado y servicio para las mujeres; y los puestos científicos y tecnológicos para los hombres (Trianes Torres et al., 2012). "Algunas jóvenes temen ser consideradas "poco femeninas" si sigue una carrera universitaria "para hombres" o si se destacan demasiado en asignaturas "masculinas" (Barmeosolo Beltrán, 2010: 397). La autolimitación por parte de las mujeres ha hecho que sus carreras profesionales no se desarrollen de la misma manera que la de los varones (Sánchez García et al., 2013). Así mismo, la elección de carrera se asocia a factores como: tradiciones familiares, al salario, o a la popularidad mediática (Santana Vega, 2015). Esto conlleva que algunos adolescentes se inclinen por profesiones prometedoras aunque no les brinde ninguna satisfacción personal (Aguirre Batzán, 1994; Llinás González, 2009).

C. El contexto escolar ha sido parte fundamental en los procesos de orientación profesional a lo largo de la historia ( Alonso, 2010; Sánchez y Álvarez, 2012). Según Bermeosolo Bertrán (2010) el rechazo y la marginación que viven muchos adolescentes debido a su género, apariencia física, rendimiento académico, etc, exigen mayor atención en los contextos escolares. Cabe resaltar que la escuela es la llamada a servir de puente entre el adolescente que se está preparando en el interior de las aulas y el sector productivo, para ello, debe generar planes, programas o proyectos de orientación escolar que faciliten esta interacción (Martín et al., 2011; Reppeto et al., 2009; Rodríguez Moreno, 2014; Santana Vega, 2015). Dentro del contexto escolar debería prestársele mayor énfasis al conocimiento de las aptitudes, intereses y motivaciones del alumnado con el fin de ayudarle en la toma de decisiones sobre su futuro académico-profesional (González-Benito y Vélaz-de-Medrano Ureta, 2014) 
D. El género -y el estereotipo de género- influye, aún hoy, en la elección profesional de los estudiantes (Barrero González, 2006; Alonso, 2010; Santana Vega et al., 2012; Jiménez Vásquez, 2013). Para Sánchez et al., (2013) la participación de la mujer en el mercado laboral ha aumentado a nivel global. Sin embargo, el género influye en forma decisiva en la vida social y laboral de los seres humanos, dado que esta condición brinda oportunidades, regularmente, a los hombres, y limitaciones o marginaciones a las mujeres (Repetto et al., 2009; Sánchez et al., 2013; Chávez y Ríos, 2014). Un ejemplo de ello es que a pesar de que las universidades registran mayor matricula y culminación de los programas académicos por parte de las mujeres, su inserción laboral sigue mostrando desventajas.

El 67\% de las mujeres están en empleos de baja productividad, asociados a una situación más precaria en términos de protección social y retribución económica. A pesar de que tienen mayores niveles de formación y capacitación, la tasa de desempleo de las mujeres es otro indicador relevante, puesto que es un 35\% mayor que la de los hombres. El ingreso promedio de las mujeres continúa siendo inferior al de los varones con márgenes de un $60 \%$ a $90 \%$ e, incluso, las mujeres más calificadas perciben menos ingresos que los hombres con su mismo nivel educacional (Rico y Trucco, 2014: 9)

Atendiendo a Rodríguez Moreno (2014) históricamente la mujer ha sido vista como incapaz o incompetente, y sus actitudes siempre han sido orientadas a que asuman un rol dependiente dentro de la sociedad y la familia. Este rol o comportamiento esperado de la mujer es el que la sociedad considera, sin duda alguna, aceptable (Rico y Trucco, 2014, cit., Correll, 2001).

Esta investigación está en la misma línea de la realizada por el grupo de investigación GIOES de la Universidad de La Laguna (Santana Vega et al., 2009, 2012, 2016; Santana Vega, Feliciano García y Santana Lorenzo 2012, 2013; Santana Vega y Feliciano García, 2009; 2011; Santana Vega et al., 2010). El objetivo de este estudio es análizar qué carrera prefieren estudiar los chicos y chicas al finalizar el bachiller. En concreto, se planteó los siguientes interrogantes: 1) ¿Qué piensan hacer los estudiantes cuando terminen su bachillerato? 2) ¿Cuáles son los motivos o intereses que los lleva a tomar su decisión o elección? 3) ¿Como incide el género en la toma de decisiones al final del bachillerato?

\section{Método}

En la investigación se llevó a cabo un estudio de carácter descriptivo, en el cual se utilizó como instrumento de recogida de datos un cuestionario diseñado ad hoc para examinar los diferentes motivos e intereses personales en el momento de seleccionar una carrera profesional.

\section{Sujeto}

Participaron 155 estudiantes (67 alumnas y 88 alumnos), lo cual corresponde a 44,1 $\%$ de mujeres y 56,9 \% de hombres, de la Institución Educativa Alfonso López Pumarejo en la ciudad de Cartago, Colombia, de carácter oficial. El alumnado se encuentra cursando octavo, noveno, décimo y undécimo y tienen en promedio 15, 5 años de edad (Tabla 1). El centro Pumarejo se seleccionó de manera intencional según los siguientes criterios: 1) 
Carecer de un programa estructurado de orientación académica y laboral. 2) El alumnado presentaba dificultades en el momento de elegir una carrera profesional

Tabla 1

Distribución del alumnado por nivel académico, con indicación de la edad promedio

\begin{tabular}{llccc} 
& & \multicolumn{3}{c}{ Edad } \\
\cline { 3 - 5 } & & $\mathrm{N}$ & Media & $\begin{array}{c}\text { Desviación } \\
\text { Típica }\end{array}$ \\
\hline \multirow{4}{*}{ Grado } & Octavo & 49 & 14,5 & 1,1 \\
& Noveno & 44 & 15,1 & 1,0 \\
Décimo & 26 & 16,1 & 1,1 \\
& Undécimo & 36 & 16,7 & 1,4 \\
& Total & 155 & 15,5 & 1,4 \\
\hline
\end{tabular}

Nota: Fuente: propia

\section{Instrumento}

El Grupo de Investigación en Orientación Educativa y Sociolaboral (GIOES) de la Universidad de la Laguna diseñó el cuestionario "Cuestionario de Orientación AcadémicoLaboral (COAL)". El cuestionario consta de 2 escalas en las que se analiza: a) los motivos e intereses personales en la elección de carrera, y b) el desarrollo de carrera. El COAL incluye una pregunta para conocer los motivos e intereses personales que tienen los estudiantes en el momento de seleccionar una profesión. La variable tiene 15 interrogantes con 4 alternativas de respuestas puntuables de 1 (nada) y 4 (mucho). El coeficiente de á de Cronbach tuvo un valor de 0.725. En cuanto a su estructura interna, la prueba KMO dio un valor de 0.664 y la prueba de esfericidad de Bartlett fue significativa ( $p<0.000$ ). La extracción de la matriz de componentes generó 5 dimensiones que explicaban el 58,8\% de la varianza total y que agrupan los motivos relativos a: F1) dinero, prestigio y estabilidad; F2) innovación; F3) altruismo; F4) facilidad/accesibilidad de los estudios; F5) intereses profesionales.

Tabla 2

Matriz de componentes rotados

\begin{tabular}{|c|c|c|c|c|c|}
\hline & \multicolumn{5}{|c|}{ Componente } \\
\hline & 1 & 2 & 3 & 4 & 5 \\
\hline Me permitirá disfrutar de nuevos retos y aventuras & ,759 & & & & \\
\hline Me permitirá participar en actividades innovadoras & ,715 & & & & \\
\hline Me permitirá no hacer siempre lo mismo en el trabajo & 649 & & & & \\
\hline Me permitirá disponer de tiempo para mis aficiones & ,439 & & & & \\
\hline Mi familia la considera adecuada para mí & & ,773 & & & \\
\hline Los estudios de esa profesión son fáciles de aprobar & & 699 & & & \\
\hline El orientador/a la considera adecuada para mí & & 675 & & & \\
\hline Me permitirá ayudar a otras personas y solucionar sus problemas & & & 817 & & \\
\hline Me permitirá mejorar el mundo en el que vivimos & & & ,787 & & \\
\hline Está relacionada con mis intereses profesionales & & & & ,693 & \\
\hline Me veo capacitado para cursar los estudios de esa profesión & & & & ,596 & \\
\hline Me permitirá ser una persona famosa e influyente & & & & ,472 & \\
\hline Tiene muchas salidas en el mercado laboral & & & & & ,814 \\
\hline Me permitirá encontrar un trabajo pronto & & & & & ,611 \\
\hline Los estudios para realizar esa profesión no requieren mucho dinero & & & & & ,406 \\
\hline
\end{tabular}

Nota: Fuente: propia 


\section{Análisis de los datos}

El análisis de la información se realizó a través del programa SPSS. 20 y comprendió: estadísticos descriptivos para cada una de las variables, fiabilidad (alfa de Cronbach), análisis factorial, análisis de varianza de un solo factor (ANOVA), contraste de medias para muestras independientes, coeficiente de correlación (Chi cuadrado, contingencia y Pearson).

\section{Procedimiento}

Para la aplicación del cuestionario se contó con el apoyo de los profesores directores de grupo de $\left(8^{\circ}\right.$ a $\left.11^{\circ}\right)$ y del coordinador de la Institución. Previamente, se había socializado con el cuerpo docente el propósito del instrumento y el papel de cada uno en el momento de la aplicación de la prueba. El cuestionario se llevó a cabo en un horario habilitado por la Institución para tal fin.

\section{Resultados}

\section{Resultado para la decisión tomada al final del bachillerato por género}

La distribución porcentual para la decisión tomada al final del bachillerato por género (figura 1), muestra que existe una relación estadísticamente significativa; el 16,3\% de los hombres y el 6,3\% de las mujeres no saben qué hacer al final del bachillerato; el $19,8 \%$ de los chicos y el 7,8\% de las chicas piensan trabajar; el 9,3\% de los muchachos y el 6,3\% de las muchachas quieren estudiar un técnico; el 5,8\% de los chicos y el 4,7\% de las chicas desean continuar sus estudios en una academia; el 31,4\% de los hombres y el $64,1 \%$ de las mujeres desean realizar una carrera universitaria; el $17,4 \%$ de los chicos y el $10,9 \%$ de las chicas poseen otras alternativas. Se destaca que el porcentaje de chicos que deciden trabajar es mayor que el de las mujeres, mientras que el porcentaje mayor que eligen iniciar una carrera universitaria está en las chicas.

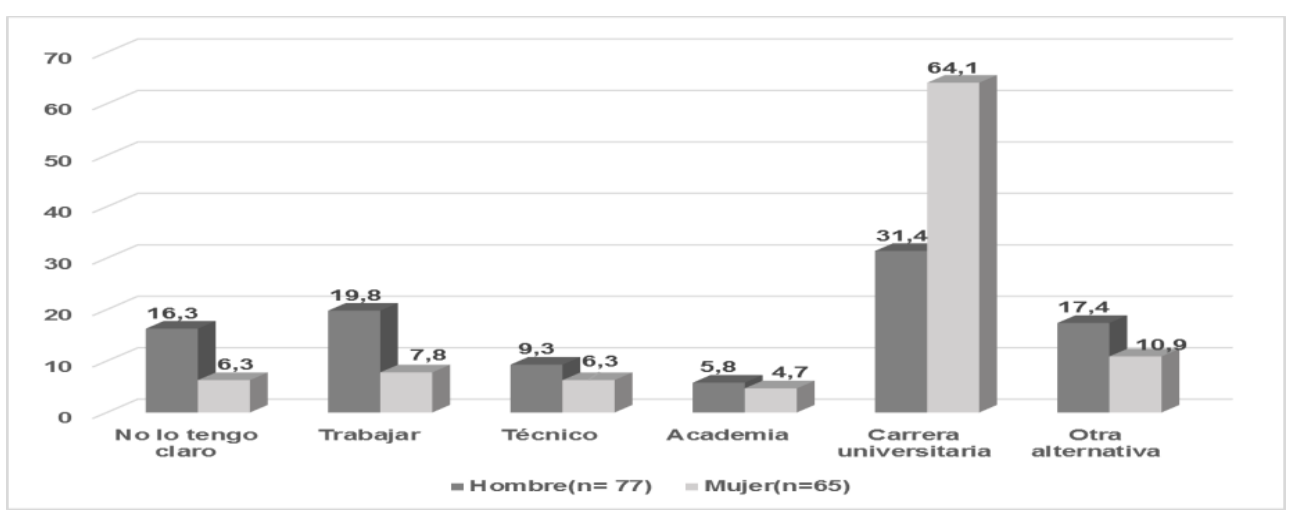

Figura 1. Distribución porcentual para la decisión tomada al final del bachillerato por género $\chi^{2}=16,862 ; \mathrm{gl}=5 ; \mathrm{p}=0,005$; coeficiente de contingencia $=0,318 ; \mathrm{p}=0,005$.

\section{Resultado para la elección de carrera por género}

La distribución de la elección de carrera por género (figura 2), es estadísticamente significativa; el 52,3\% de las mujeres tienen una mayor preferencia por las ciencias de la salud, como (Medicina, Odontología, Ciencia Forenses, Fisioterapia, Zootecnia), frente al 
$19,5 \%$ de los hombres; el 23, $4 \%$ de los chicos y el 1,5\% de las chicas prefieren hacer un técnico (auxiliar de vuelo, mecánica dental, auxiliar de enfermería); el 12,3\% de chicas y el $5,2 \%$ de chicos se inclinan por la ciencia de la educación y humanidades (Comunicación, Ed. Física, Pedagogía) el 9,1\% de los chicos y el 10,8\% de las chicas prefieren estudiar ciencias sociales y administración (contaduría, derecho, negocios internacionales); el 2,6\% de los hombres y el 4,6\% de las mujeres expresan vocación hacia las artes (diseño gráfico, actuación, modelaje, etc.); el 31,2\% de los hombres y el 10,8\% de las mujeres manifiestan inclinar su vocación hacia la ingeniería y la arquitectura (mecatrónica, mecánica industrial); el 9,1\% de los chicos y el 7,7\% de las chicas tienen como opción las fuerzas armadas. Las chicas eligen como primera opción las profesiones de carácter asistencial con el 52,3\%, y los chicos la ingeniería y la arquitectura, con el $31,2 \%$.

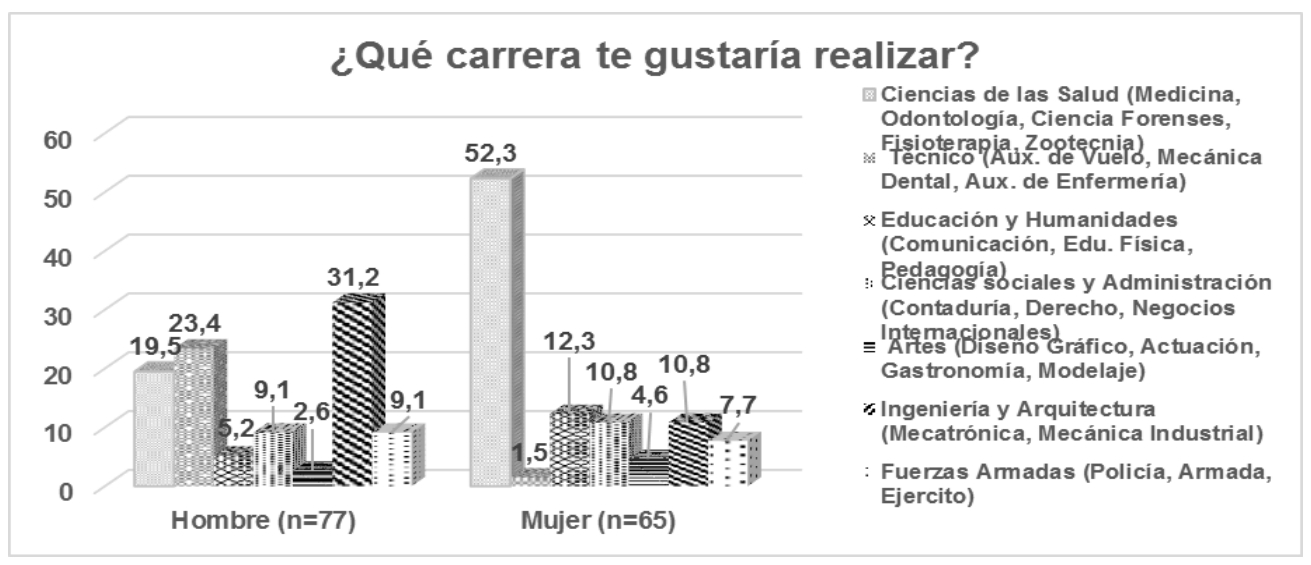

Figura 2. Si en el futuro haces estudios universitarios ¿qué carrera te gustaría realizar?- $\chi 2$ $=32,989 ; \mathrm{gl}=6 ; \mathrm{p}<0,000 ;$ coeficiente de contingencia $=0,434 ; \mathrm{p}<0,000$

\section{Discusiones y conclusión}

Una vez finalizada la investigación, se puede concluir que:

Con respecto a la elección de carrera se halló la tendencia social encontrada en investigaciones previas (Domínguez Blanco, 2004; Riquelme, Pacenza y Herger, 2008; Eurydice, 2010; PISA, 2012; Rico y Trucco, 2014; el Instituto Nacional de las Mujeres en México, 2015; Ministerio de Educación en Chile, 2015; García Ramírez, 2016; Rodríguez Méndez, Peña Calvo, y García Pérez, 2016); en tales trabajos se aprecia que las mujeres se decantan por profesiones de carácter asistencial (medicina, odontología y fisioterapia), seguidas por las licenciaturas en educación y las humanidades; mientras los hombres tienen una gran tendencia por las ingenierías y la arquitectura, seguidas por los estudios técnicos laborales, como son: mecánica dental, y auxiliar de vuelo. El análisis muestra una estrecha relación con el estereotipo sexista heredado por generaciones, en el que la mujer hace su elección de carrera llevada más por la sensibilidad natural que la caracteriza, y que se manifiesta en un deseo, constante, de querer cooperar socialmente con las demás personas $\mathrm{y}$ al entorno en el que habitan; mientras el hombre toma su decisión como una manifestación de su habilidad física, expresando gran preferencia por los trabajos que demanden esfuerzo corporal (Ruiz Pinto, García Pérez, y Rebollo- Catalán, 2013; Santana Vega et al., 2009; Santana Vega, et al., 2013; Cubillas et al., 2016) quizás como una respuesta a su virilidad. 
Sin embargo, a pesar de la feminización y masculinización de las profesiones, en la actualidad, las mujeres han accedido a diferentes sectores laborales de la sociedad (Morejón Sabio, 2010). Así mismo, entre los motivos que tienen los hombres para seleccionar una carrera es que buscan ser, más rápidamente, independientes a nivel económico, obtener el reconocimiento de los demás a través de la fama o el prestigio (Rovella, Sans de Uhrlandt, Solares, Delfino, y Diaz, 2008). De acuerdo con Rico y Trucco (2014) las mujeres Latinoamericanas se encuentran en desigualdad de condiciones ya que siempre se encasillan en profesiones menos redituables, con bajo prestigio social y que ofrecen pocas oportunidades de acceder a cargos de mayor jerarquía y mejor remunerados. Gámez y Marrero (2003); Merino Pantoja, Morong Reyes, Arellano Araya, y Merino Vidangossy (2015) denominan al instinto natural de hacer su elección a partir del reconocimiento de sus propias capacidades, gustos e intereses, motivación intrínseca. Para Morejón Sabio (2014) prestigio social, poder, reconocimiento social, y prosperidad económica forman parte de; criterios extrínsecos.

La cultura patriarcal ha promovido, entre otras cuestiones, la asociación de la afectividad -el corazón- con las mujeres y de la inteligencia -la razón- con los hombres. La socialización diferencial de género (Subirats y Brullet, 1988) va determinando, pues, el comportamiento y las expectativas de ambos sexos en todos los órdenes de la vida. La mujer históricamente se ha visto atrapada en una sutil tela de araña que se ha estado entretejiendo en torno al mundo femenino: su papel en la sociedad se ha circunscrito al entorno familiar, o bien cuando accedía a la esfera pública su papel era sistemáticamente devaluado. De ahí que la mujer haya tenido serias dificultades para reconocerse y reconocer a otras desempeñando ocupaciones distintas a su ya tradicional condición de ama de casa. Pero qué duda cabe que las mujeres son y representan la mitad de la humanidad y la otra mitad de la experiencia humana y, como tales, reclaman su participación activa en la construcción del mundo.

\section{Referencias}

Eurydice. (2009). Diferencia de género en los resultados educativos: medidad adoptadas y situación actual en Europa, pp. 1-145, doi 10.2797/48598.

Aguirre Batzán, Á. (1994). Psicología de la adolescencia. Barcelona: Boixareu Universitaria.

Alonso A., J. (2010). Manual de orientación educativa y tutoría Educación media y media superior. México: Laza y Valdés.

Arancibia C., V., Herrera P., P., y Strasser S., K. (2008). Manual de Psicología educacional, sexta edición. Chile: Universidad Católica.

Blanco García, N. (1999). ¿De qué mujeres y de qué hombres hablan los libros de texto? Kikirikí. Cooperación Educativa, 54, pp. 47-52.

Barrero González, N. (2006). Manual para el diseño y evaluación de los programas de orientación educativa. Argentina: El Cid.

Bermeosolo Bertrán, J. (2010). Psicopedagogía de la diversidad en el aula. Desafío a las barreras en el aprendizaje y la participación. México: Alfaomega.

Bisquerra Alzina, R. (1992). Orientación psicopedagógica para la prevención y el desarrollo. Barcelona: Boixareu Universitaria. 
Castells, M. (1998). La era de la información. Economía, sociedad y cultura. El poder de la identidad (Vol.2). Madrid: Alianza.

Chávez, N. M., y Rios, H. (2014). Discriminación salarial por género: Efecto techo de cristal. Caso: siete áreas metropolitanas de Colombia. Revista Dimensión Empresarial. Vol. 12 (2), pp. 29-45. Recuperado de http://www.uac.edu.co/images/stories/publicaciones/revistas_cientificas/dimensionempresarial/volumen-12

Cubillas Rodríguez, M., Abril Valdez, E., Domínguez Ibáñez, S., Román Pérez, R., Hernández Montaño, A., y Zapata Salazar, J. (2016). Creencias sobre estereotipos de género de jóvenes universitarios del norte de México. Revista Perspectivas psicológicas, Vol. 12 (2), pp. 217-230. Recuperado de http://www.scielo.org.co/pdf/dpp/v12n2/v12n2a05.pdf.

Domínguez Blanco, M. (2004). Equidad de género y diversidad en la educacion colombiana. Revista Electrónica de Educación y Psicología- REPES-, 2, pp. 1-19. Recuperado de http://Revistas.Utp.Edu.Co/Index.Php/Repes/Article/View/5205.

Gámez, E. y Marrero, H. (2003) Metas y motivos en la elección de la carrera de psicología. Revista Anales de Psicología, vol 19 (1), pp. 121-131. Recuperado de https://www.researchgate.net/publication/28076476.

García Ramírez, C. T. (2016). Contribuciones de la Teoría Social Feminista y los Estudios de Género a la sociología Venezolana. Revista Espacio Abierto: cuaderno venezolano de socilogía, 25 (4) pp. 47-60. Recuperado de http://www.redalyc.org/articulo.oa?id=12249087004.

Gaviria, J.L. (1994). El sexo y la clase social como determinantes de los intereses profesionales en el último curso de secundaria. Revista de Investigación Educativa, $23,262-273$.

Gimeno Sacristán, J. (1998). Poderes inestables en educación. Madrid: Morata.

González- Benito, A., y Velaz- de- Medrano Ureta, C. (2014). La acción tutorial en el sistema escolar. Madrid: UNED.

Programa de las Naciones Unidas. (2016). Programa de las Naciones Unidas para el Desarrollo en Colombia. Recuperado de http://www.co.undp.org/content/colombia/es/home/post2015/mdgoverview/overview/mdg3/

Imbernón, F. (1999). Amplitud y profundidad de la mirada. La educación ayer, hoy y mañana. En Imbernón, F. (Coord.). La educación en el siglo XXI. Retos del futuro inmediato (pp.63-79). Barcelona: Graó.

Instituto Nacional de Las Mujeres en México. (2015). El impacto de los estereotipos y los roles de género en México. Centro de documentación turística. Recuperado de http://cedoc.inmujeres.gob.mx/documentos_download/100893.pdf.

Jiménez Vásquez (Coord.), M. S. (2013). La tutoría en la escuela secundaria la visión de sus actores en escuelas técnicas de tlaxcala. México: Díaz de Santos.

Llinás González, E. (2009). La orientación académica desde el bienestar universitario. Barranquilla: Uninorte.

López Sáez, M. (1995). La elección de una carrera típicamente femenina o masculina desde una perspectiva psicosocial: la influencia del género. Madrid: CIDE. 
Martín, E., Solé, I., Almirall, R., Babío, M., Bassedas, E., Castells, N., y Vélaz de Medrano, C. (2011). Orientación educativa: Modelos y estrategias de intervención. Barcelona: Grao.

Mérida Serrano, R., Ramírez García, A., Corpas Reina, C., y González Alfaya, M. (2012). La orientación en Educación Infantil: Una alianza entre los agentes educativos. Madrid: Pirámide.

Merino Pantoja, E., Morong Reyes, G., Arellano Araya, A. O., y Merino Vidangossy, E. (2015). Características, motivaciones y expectativas de estudiantes de género masculino de carreras pedagógicas de la universidad Bernardo O`higgins. Revista Electrónica Actualidades Investigativas en Educación, vol. 15(3), 1-24. Recuperado de http://www.redalyc.org/articulo.oa?id=44741347009.

Ministerio de Educación de Chile. (2015). Educación para la igualdad de género: Plan 2015-2018. Recuperado de http://www.mineduc.cl/wpcontent/uploads/sites/19/2017/01/CartillaUEG.pdf.

Ministerio de Educación Nacional. (2013). Resultados de las condiciones laborales de los graduados de educación superior 2001-2012 y los certificados de educación para el trabajo y el desarrollo humano 2010-2012 (Documento Técnico). Recuperado de http://www.graduadoscolombia.edu.co/html/1732/articles334303_documento_tecnico_2013.pdf.

Morejón Sabio, R. (2010). Género, autoeficacia y proyección profesional: La transición universidad- mundo laboral.Bilbao: Instituto Vasco De La Mujer. Recuperado de http://www.emakunde.euskadi.eus/contenidos/informacion/publicaciones_subvenci onadas/es def/adjuntos/902E-Genero-Autoeficacia.pdf.

Organización de las Naciones Unidas para la Educación, la Ciencia y la Cultura. (2012). Matriculación y género: tendencia en los estudios superiores. Atlas Mundial de la igualdad de género en la educación, pp. 74-85. Recuperado de http://unesdoc.unesco.org/Ulis/cgi-bin/ulis.pl?catno=217311\&gp=0\&lin=1\&ll=3

PISA (2012). ¿A qué tipo de carreras anspiran los chicos y las chicas? Recuperado de https://www.oecd.org/pisa/pisaproducts/pisainfocus/PISA\%20in\%20Focusn\%C2\%B014\%20ESP.pdf.

Repetto , E.(Dir.)., Guillamón, J., Malik, B., Vélaz de Medrano, C., Moreno, J., y Téllez, J., Mesa, L. (2009). Modelos de orientación e intervención psicopedagógica: intervenciones psicopedagógicas para el desarrollo del aprendizaje, de la carrera $y$ de la persona. Madrid: UNED.

Rico, M., y Trucco, D. (2014). Adolescentes: Derecho a la educación y al bienestar futuro. CEPAL, pp. 1-91. http://www.cepal.org/es/publicaciones/35950-adolescentesderecho-la-educacion-al-bienestar-futuro.

Riquelme, G., Pacenza, M., y Herger, N. (2008). La universidad frente a las demandas sociales y productivas. Estudio y trabajo de estudiantes universitarios: acceso al empleo, etapas ocupacionales y expectativas sobre la vida profesional. Tomo II. Argentina: Miño y Dávila.

Rivière, M. (2000). El mundo según las mujeres. Madrid: El País-Aguilar.

Rodríguez Méndez, M., Peña Calvo, J. V., y García Pérez, O. (2016). Estudio cualitativo de las diferencias de género en la elección de opciones académicas en los 
estudiantes del bachillerato científico-técnico. Revista Usal, 28 (1), pp. 189-207, doi: http://dx.doi.org/10.14201.

Rodríguez Moreno, M. (2014). Cómo orientar hacia la construcción del proyecto profesional: Autonomía individual, sistema de valores e identidad laboral de los jóvenes. Bilbao: Desclée de Brouwer.

Rovella, A., Sans de Uhrlandt, M., Solares, E., Delfino, D., y Diaz, D. (2008). Motivo de logro y elección de la carrera de psicología. Revista Electrónica de Psicología Política, 18, pp. 93-100. Recuperado de http://www.psicopol.unsl.edu.ar/Diciembre2008_Nota5.pdf.

Ruiz Pinto, E., García Pérez, R., y Rebollo- Catalán, Á. (2013). Relaciones de género de adolescentes en contextos educativos. Análisis de redes sociales con perspectiva de género. Revista de Curriculum y Formación del Profesorado, Vol. 17(1), pp. 123140. Recuperado de https://dialnet.unirioja.es/servlet/articulo?codigo=4334991.

Sánchez García, M., Suárez Ortega, M., Padilla Carmona, M., Bisquerra Alzina, R., Baelo Álvarez, R., Martínez García, I., y Moreno Calvo, A. (2013). Orientación profesional y personal. Madrid: UNED.

Sánchez, M. F. (Coord.), y Alvárez, M. (2012). Bases teórico-prácticas de la orientación profesional. Madrid: Uned.

Santana Vega, L. E. (2015, 4a ed.). Orientación educativa e intervención psicopedagógica. Cambian los tiempos, cambian las responsabilidades profesionales. Madrid: Piramide.

Santana Vega, L. E. (2002). Mujeres, igualdad de oportunidades y transición sociolaboral. Revista de Educación, 327, pp. 169-187. Recuperado de https://www.mecd.gob.es/dctm/revista-deeducacion/articulos327/re3271110520.pdf?documentId=0901e72b812598b2

Santana, L.E., Feliciano, L. y Jiménez, A.B. (2016). Perceived family support and the life design of immigrant pupils in secondary education. Revista de Educación, 372, pp. 35-62. doi: 10.4438/1988-592X-RE-2015-372-314

Santana Vega, L. E. y Feliciano García, L. y Santana Lorenzo, A. (2013). Madurez y autoeficacia vocacional en $3^{\circ}$ y $4^{\circ}$ de la ESO, Bachillerato y Ciclos Formativos. Una perspectiva de género. Revista Española de Orientación y Psicopedagogía, 24(3), pp. 8-26. Recuperado de http://www.uned.es/reop/pdfs/2013/24-3\%20\%20Santana.pdf

Santana Vega, L. E.; Feliciano García, L. y Jiménez Llanos, A. B. (2012). Toma de decisiones y Género en Bachillerato, Revista de Educación, 359, pp. 357-387, doi: 10.4438/1988-592X-RE-2011-359-098

Santana Vega, L. E. y Feliciano García, L. Santana Lorenzo, A (2012). Análisis del proyecto de vida del alumnado de educación secundaria, Revista Española de Orientación y Psicopedagogía, 23 (1), pp. 26-38. Recuperado de http://www.uned.es/reop/pdfs/2012/23-1\%20-\%20Santana.pdf

Santana Vega, L. E. y Feliciano García, L. (2011). Percepción de apoyo de padres y profesores, autoconcepto académico y toma de decisiones en Bachillerato, Revista de Educación, 355, pp. 493-519. Recuperado de http://www.revistaeducacion.educacion.es/re355_21.html 
Santana Vega, L. E.; Feliciano García, L. y Cruz González, A. (2010). El Programa de Orientación Educativa y Sociolaboral: un instrumento para facilitar la toma de decisiones en educación secundaria. Revista de Educación, 351, pp. 73 -105. Recuperado de http://www.mecd.gob.es/revista-de-educacion/numeros-revistaeducacion/numeros-anteriores/2010/re351/re351_04.html

Santana Vega, L. E. y Feliciano García, L. (2009). Dificultades en el proceso de toma de decisiones académico-profesionales: el reto de repensar la orientación en Bachillerato. Revista de Educación, 350, pp. 323-350. Recuperado de http://www.revistaeducacion.educacion.es/re350/re350.pdf

Santana Vega, L. E., Feliciano García, L. Jiménez Llanos, A.B. (2009). Autoconcepto académico y toma de decisiones en el alumnado de Bachillerato. Revista Española de Orientación y Psicopedagogía, 20(1), pp. 61-75. Recuperado de http://www.uned.es/reop/pdfs/2009/20-1\%20-\%20Lidia\%20Santana.pdf

Subirats, M.; Brullet, C. (1988). Rosa y azul. La transmisión de los géneros en la escuela mixta. Madrid: Instituto de la Mujer.

Torres Santomé, J. (1994): Globalización e interdisciplinariedad: el curriculum integrado. Madrid: Morata.

Trianes Torres, M. V., Amezcua Membrilla, J. A., Barajas, C., Bendayan, R., Berben, A., Cano, F., y Sánchez Sánchez, A. (2012). Psicología del desarrollo y de la educación. Madrid: Pirámide.

Fecha de recepción: 22/06/2017

Fecha de revisión: 02/10/2017

Fecha de aceptación: 16/11/2017 